\title{
Trend of Random Events in Organizing When Influenced by a Non-Observable Factor
}

\author{
Charles Roberto Telles \\ Grupo Administrativo Setorial, Secretary of State for Education of Paraná, Curitiba, Brazil \\ Email: charlestelles@seed.pr.gov.br
}

How to cite this paper: Telles, C.R. (2018) Trend of Random Events in Organizing When Influenced by a Non-Observable Factor. Open Journal of Statistics, 8, 85-93. https://doi.org/10.4236/ojs.2018.81007

Received: December 15, 2017

Accepted: February 2, 2018

Published: February 5, 2018

Copyright $\odot 2018$ by author and Scientific Research Publishing Inc. This work is licensed under the Creative Commons Attribution International License (CC BY 4.0).

http://creativecommons.org/licenses/by/4.0/

\begin{abstract}
This article analyzes in complex systems the web of variables that constitute the formation and behavior of an event organizing it in several probabilities. Based on the current statistical methodologies, multifactorial analysis associated with fuzzy logic, complex phenomena are analyzed by stating the influence of a variable to others and possibly indicating how complexity works. However, these analyzes have limitations regarding the scope of the samples considering the mechanics of an event determined only by the non-physical quantitative properties of variables. The mentioned limits, refers to not considering the measurement of the variable's interactions influence in the event by analyzing the frequencies in which the interactions affect the formation and behavior of the expected event. Considering that interactions take place in the physical world, they can present non-observable physical features that influence the event. This observation can point out the periodic function in the production of the complex events that can be observed considering the frequency with which the analyzed event occurs in its physical quantitative characteristics.
\end{abstract}

\section{Keywords}

Complexity, Statistics, Statistical Physics, Pattern Formation

\section{Introduction}

The main characteristic of the phenomena approached for the methodology to be explained in this article is to deal with events whose variations in being and previous formation and behavior of the event is the result of a tangle of other events (coupling functions [1]) associated with it [2]. The variables that promote 
the event may have many expressions, generating oscillations in results [3]. The analysis of this article refers to the measurement of the frequencies of interaction among variables (dynamical systems) and the influence of these frequencies on the formation of the expected event. The expression frequency of interaction among variables implies in the idea that forming events (variables) of the main event and their behavior can interact with each other influenced much more by frequency in which interactions between matter and energy express regarding a quantitative physical aspect, possibly changing the results of expression in the event. So, it is aimed to point out how the frequency with which interactions among those variables occur under the concept of a multifactorial statistical analysis associated with Fuzzy Logic [4] and subsequent observation of the parameter space of the variables chained together [5]. When analyzing a complex event, the interaction among variables is not always easily or possibly observable or reproducible, and those interactions differ in its expressions generating to the experiment or observation, oscillations. Conceiving the event as being determined by frequency of interactions prevents the flow of the event from being observed only from the view of nature possibilities described as a parameter space of the event, which naturally don't occur in this way in the raw state of nature [5] [6] [7]. Therefore, the results from statistical surveys that delimit the object of study by the parameter space are questioned, even if they consist of Fuzzy-like parameters, which, in this article, is extended by the analysis of variables and their correlation with the complex system of the event considering all the interactions among variables of a given event and the power of influence of these interactions in the system, possibly causing a periodicity or coupling functions [1] [6] [8].

The investigation of events that have variables with non-binary analysis parameters (fuzziness) becomes necessary to the extent that facts involving these phenomena become uncontrolled due to the large range of factors that cause a particular event to occur [9], such as, the great cities and the integration between human being and nature, or the phenomena that have in their structure physical, chemical or biological dimensions within it [1] [10]. In this way it is necessary to delimit in the statistical methods the feasibility in interfering in the phenomenon identifying which parameters, their variances and quantitative physical characteristics are most influential within the system. In traditional analyzes, the samples in a multifactorial statistical analysis associated with Fuzzy Logic have scopes in which there is a wide variance distribution of the analyzed variables [4] [11], however, the internal movement that occurs between one variable and another, in terms of its interactions is not observed, but by the final sample data set and the use of mathematical tools that do not explain the relation between the parts of the samples from that point of view [12]. This form of analysis is not sufficient to visualize cause and effect relationships in the entanglement of event variables and their dynamics in modifying in time [13] when taking in account the matter and energy quantitative physical aspects of influence. 


\section{Fundamental Principles}

\subsection{Interactions Frequency}

Nowadays the main objective is to understand ways of considering large number of variables in statistical analysis and the influence in which variables have among themselves (phenomena behavior). But more than the number of variables or the parameter space that figures out the aspect of nature possibilities [5], it is necessary to understand the frequency in which variables interacts with each other. Interactions can't be seen only by extracting several experimentations and statistical results. It is assumed that the quantitative physical properties of nature may have specific mechanics and they affect the experiment and could be in the search in actual statistics for a mechanics in complex events [13] [14] [15] [16].

Traditional methodological procedures of non-linear statistics verify only a portion of the state of nature of the event [12]. Statistical models that analyze complex events usually bring to light, indicators determined to give the researcher the global view of a system in a functional state of complexity restricted to a certain space and time [13]. It can happen of correspondences among analyzed variables, results and the samples, share oscillations that are caused and influenced by frequencies of variables interaction in the event, leading to a nonobservable factor. Far from considering an analysis about interactions frequency in events only related to understand the phenomena by its causes, it is meant to investigate the matter/energy properties of occupy a physical space for example, or other quantitative physical aspects in the events and how the physical aspects are influenced by the amount of interactions in the phenomena modifying objective aspects of known cause and effect. Frequency can lead to a concept of proportionality between cause and effect as result of number of interactions in which variables share [1] [9] [14] [15] [17] [18].

The discussion proposed in this paper has as its basic premise the idea that the oscillations in an event tend to be delimitated by an unobservable factor, and must have an organization in which the probable is set to happen towards any sort of the given possibilities based on the quantitative physical aspects of the event. In theory the description refers to the possibility that in complex systems, variables exert influences on one another by the number of interactions they exert, considering aspects mentioned before as spatial, temporal, functional physical properties of objects investigated in a given complex system [5]. The unobservable factor is that possibility of internal movements (physical quantitative aspects) in the organization of the event influence the formation or behavior of the same generating a periodicity in the system rather than theoretical data expressing randomness. One example of variables quantitative physical aspects is towards Lorenz Deterministic Nonperiodic Flow experiments, where on table 1 and 2 [6], the iterations are considered under the aspect of parameter space of variables rather than the amount of particles involved in the fluid of which could exert mechanical functions in the system prior to the results [6] or with another 
point of view, space, time and other physical characteristics of an fluid could exert influence on the event behavior rather than numerical simulations [14].

It is understood that each variable has its expression, influencing a system in a gradual way, which in turn, interacts with other variables in its own gradual flows equally. Thus each chaining of variables in periodicity is an extension of the fuzzy and multifactorial aspect of nature's physical quantitative aspects [11].

\subsection{Periodicity}

The possibility of predicting events with periodicity or expressions of temporal cause and effect is dependent on the analysis of the event occurring taking into account the oscillations among non-linear variables [6].

In a multifactor analysis model case, the relationship between the presences of certain types of insects influenced by the presence of factors such as soil moisture [19] is observed. This variation of moisture reflects the presence or absence of certain types of organisms. However, other unobserved latent factors are not considered in the analysis, so the scope of sampling as well as the size of the samples, are restricted at the level of physical space of that event in occurrence and a model of nature. A physical quantitative point of view of elements in the system aren't considered as factor who could modify the behavior of the event. A garden does not reflect a wider geographic region, to which it is possible to find periodicity of the event in occur, as well as, the correspondence between the presence and absence of variables physical interactions in a sample is not sufficient to indicate causality in the event, but just a glance of it [10]. If the estimated results in a garden for only that site are considered as objective, these results have their validity restricted only to this scope of samples and yet it does not reflect an evolution in time considering a large scale phenomena of the Universe and complexity [13] [18] [20] [21] [22] [23]. More than smaller results, it would be true to assume a natural order in variables interactions in an universal way where the probable expressions in the example given can have oscillations caused much more by the interactions frequency of variables rather than the number or parameter space of variables. Or in other words, one may ask "Why results vary in each sample or clusters of samples of the same phenomena?", and the answer could be "Because there are too many variables in the system" or an alternative answer is "It depends on the frequency of interactions in which each variable express itself".

For this reason it is understood that it is necessary to widen the scope of analysis in order to obtain valid results for situations other than the investigated phenomenon, or in other words, to observe the phenomenon as a large-scale system [9] both in the possibility of variables expressions (also latent ones) as well as the probable interactions of variables that there exists unveiling periodicity for just a few factors or as for all. For the term periodicity is meant a proportionality of cause and effect among variables of a complex system given by frequency of variable's interactions. So one may assume that the cause and effect 
concept in this sense is interpreted by means of proportionality rather than determinism, relativity or uncertainty. It can be seen that the idea of order and disorder of nonlinear mechanics can be attributed to various aspects of nature [18]. In most cases, it is possible to identify the concept of proportion among variables, in their own attributes, as creators of probabilities in the phenomenon. Proportion of cause and effect can be identified in a finite space system in which the frequencies of variable's interactions may alter the results of event' probability rather than considering direct cause and effect excluded from quantitative physical aspects of variable's interactions. In the case of a broader analysis, it is possible to obtain universal frequency indicators that strongly influence a complex system for all possible situations [20] [24].

The mechanistic function in the production of the complex events can be observed considering the frequency with which the analyzed variable occurs in its quantitative interactions in relation to each one of the variables that constitute the dynamic system among them. It should be considered that any dependent or independent variable are susceptible of inter changes influenced one by other and also quantity presence of the variable in the given circumstance, such as the law of large numbers behavior. For example, if the population factor affects the consumption of water in school buildings, the share of force exerted by the population on water consumption is related not only to the size of the school population, but also to the frequency with which organisms consume the water resources influenced by other factors internal and external to the organism. It is possible that larger populations always present a higher frequency of occurrence of the event as opposed to smaller populations due to the quantity of elements that share interactions in the event, however, it is not excluded the possibility that smaller populations may present more frequency than larger ones being influenced by other factors [25]. In this sense, frequencies of one variable may be affected by other variables and it is sought to establish frequency relations between the factor analyzed in relation to the universe of factors present in a given event in order to observe how the variances of the factors (variables) interact with each other, as opposed to only verifying the frequency with which there is logical correspondence between types/number of variables and parameters analyzed [18] [22]. Events that occur by flows of quantitative frequencies of interactions in variables are necessarily unstable in their initial and final conditions, which suggests the need for more precise methodologies to identify a physical mechanics in the midst of apparent disorder [1] [4] [13] [14].

\section{Experiment Testing}

When aggregating samples, it is possible to verify the behavior of the events when they are influenced strongly or weakly by the system as a whole [26]. It means to enlarge the scope of event as well as observe the effect of latent variables and the quantitative and physical aspects of variables that is a natural condition in nature.

Proposed model: The graph below shows a variable being analyzed in its influ- 
ence in relation to all samples of the system. What is observed is the visualization of the fuzzy effect by the bands (blue line) in the variable in question (Figure 1) and the effect of a variable in relation to the whole system. The effect of verifying the interaction among variables in this way allows detecting the unobservable factor in their behavior of periodicity caused by frequency. The chart below represents only one variable under analysis. More analysis would be needed to cross data.

Note in red (Figure 2), it is observed that even if a school has large population, they don't share the same consume on water, expressing high oscillation among them. The frequency ratio caused by variables quantitative aspects has as tasks of methodological procedures as the following:

1) Consider oscillation of samples as effect of frequency of variable's interactions;

2) Scope of analysis not limited (reductionist) by number of samples and latent variables;

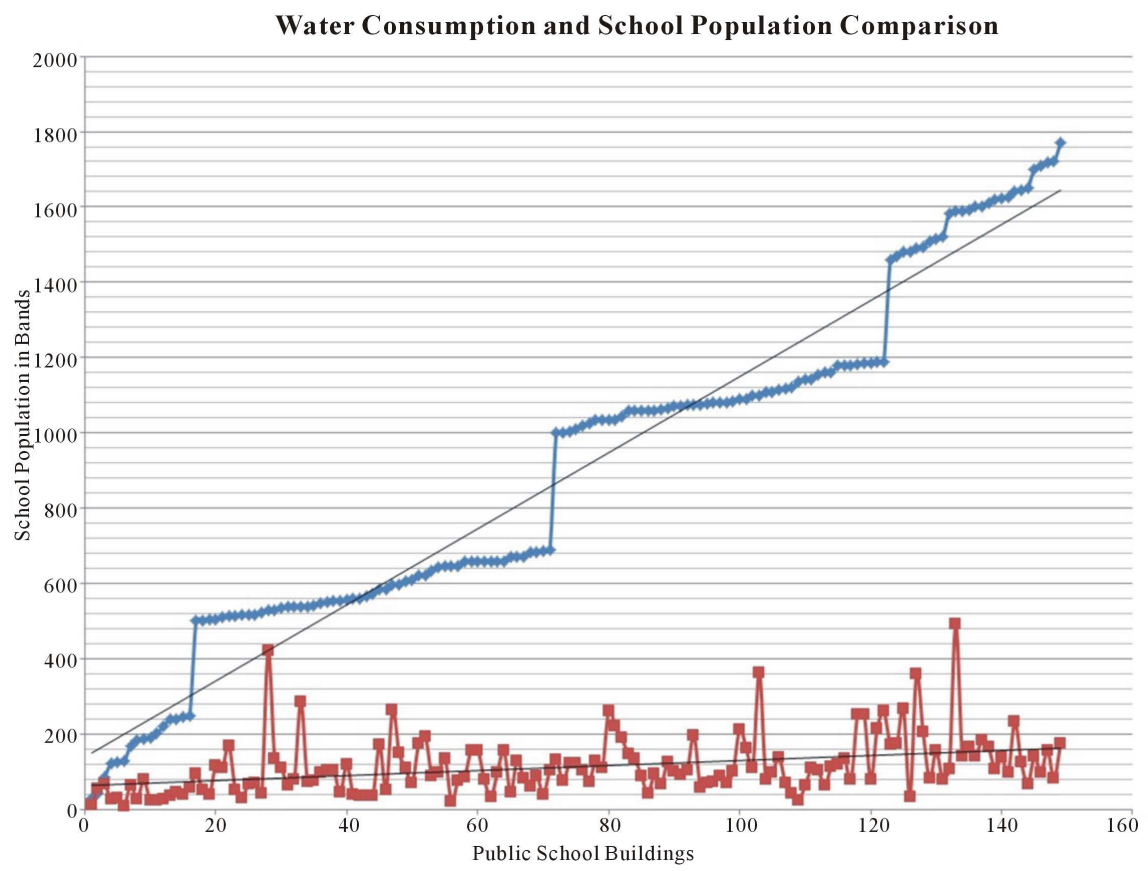

Figure 1. Analysis of the influence of school population on water consumption in relation to the universe of system variables. Axis $\mathrm{x}$-samples of educational institutions. Axis y-Population bands [25].

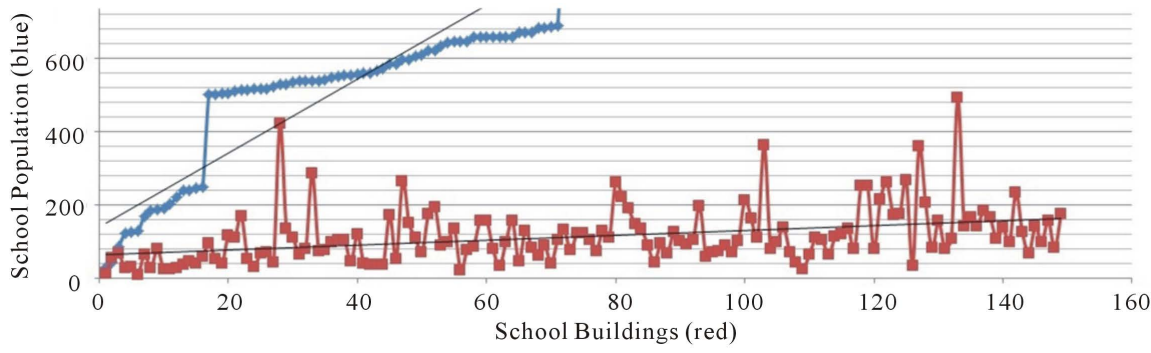

Figure 2. Highlight at axis x of Figure 1. 
3) Large number of variables;

4) The system evolve in time showing many expressions;

5) The mains task would be to measure the quantitative and physical relations among variables, that being empirical analysis rather than only theoretical;

6) Understand periodicity caused by frequency ratio in complex systems.

In Figure 1, it is observed how population of students, teachers, and others (staff) in a building school consumes water [25]. There are high oscillations of consume ( $\mathrm{x}$ axis) in the same population range (y axis bands). The graph shows that population don't influence strongly the water consumption at schools. It has a slight influence. Several other latent variables have influence in the whole causing most of the oscillations in axis $\mathrm{x}$ [25]. However, it is not possible to assume as false the possibility of the opposite of this to happen. The degree of influence of school's population on water consumption might be in periodicity due to population quantitative view aspect caused by frequency of interactions and in a larger scope, it can be seen that this type of systems reveals that other frequencies of variables interactions affects the formation and behavior of the event, and even if some institutions have low frequency of population variable interactions frequency of consuming water happening in the high band of analysis (3rd upper arrow, Figure 1), all the oscillations are caused much more by the quantitative physical aspect of population interactions among other variables in the system than an objective or theoretically view that the more population the higher the consume. Modifying a frequency can lead to proportionality terms, not being necessary to perform an experiment with restricted data set or initial conditions. Proportionality reveals itself and turns to be periodic.

\section{Conclusion}

The interactions among variables in a complex system can indicate periodicity that can evidence the "mechanical" behavior in complexity, or in other words, it is suggested that a causal relation among variables can occur under the concept of proportionality caused by frequency of interactions expressing internal movements in a phenomenon. Periodicities can occur under the physical, quantitative and indicative temporal aspects as well as the specific physical properties (functions) of the analyzed variable. Indicators of physical aspect can turn into frequency on the variable's interactions that can design mechanics behaviors or modifications among the variables in their multidimensional strings allowing the visualization of states of distribution of the variables among themselves and obtaining a constitutive model of the phenomena in organizing itself.

\section{References}

[1] della Volpe, G. (1984) A lógica como ciência histórica. Edições, Lisboa, 313 p.

[2] Butterfield, J. and Pagonis, C. (1999) From Physics to Philosophy. Cambridge University Press, Cambridge, $235 \mathrm{p}$.

[3] Treier, S. and Jackman, S. (2008) Democracy as a Latent Variable. American Journal 
of Political Science, 52, 201-217. https://doi.org/10.1111/j.1540-5907.2007.00308.x

[4] Nature (2017) Statistics for Biologists. Macmillan Publishers Limited, Springer. https://www.nature.com/collections/qghhqm/pointsofsignificance

[5] Li, C. (2012) Latent Variable Modeling. Shanghai Archives of Psychiatry, 24, 118120.

[6] Lorenz, E.N. (1963) Deterministic Non-Periodic Flow. Journal of the Atmospheric Sciences, 20, 130-141. https://doi.org/10.1175/1520-0469(1963)020<0130:DNF>2.0.CO;2

[7] NCBI. National Center for Biotechnology Information. A Simple Guide to Chaos and Complexity. http://www.ncbi.nlm.nih.gov/pmc/articles/PMC2465602/

[8] Bitar, S.D., et al. (2016) Applying Fuzzy Logic to Estimate the Parameters of the Length-Weight Relationship. Brazilian Journal of Biology, 76.

[9] Wilcox, Rand R. Phys.Org. (2017) New Statistical Methods Would Let Researchers Deal with Data in Better, More Robust Ways. The Conversation. https://phys.org/news/2017-05-statistical-methods-robust-ways.html\#jCp

[10] Wilcox, R.R. and Keselman, H.J. (2012) Modern Regression Methods That Can Substantially Increase Power and Provide a More Accurate Understanding of Associations. European Journal of Personality, 26, 165-174. https://doi.org/10.1002/per.860

[11] Martens, M. and Winckler, B. (2014) Physical Measures for Infinitely Renormalizable Lorenz Maps. ArXiv: 1412.8041v1.

[12] Prazak, M. (1972) Language and Logic. Greenwood, Westport, CT, 154 p.

[13] Stankovski, T., et al. (2017) Coupling Functions: Universal Insights into Dynamical Interaction Mechanisms. Reviews of Modern Physics, 89, 045001.

[14] Nakamori, Y. and Ryoke, M. (1999) Modeling of Fuzziness in Multivariate Data Analysis. IEEE SMC 99 Conference Proceedings of Systems, Man, and Cybernetics, 12-15 October 1999. https://doi.org/10.1109/ICSMC.1999.815565

[15] Altman, N. and Krzywinski, M. (2015) Points of Significance: Association, Correlation and Causation. Nature Methods, 12, 899-900. https://doi.org/10.1038/nmeth.3587

[16] Graça, D.S., Rojas, C. and Zhong, N. (2017) Computing Geometric Lorenz Attractors with Arbitrary Precision. Transactions of the American Mathematical Society.

[17] Vandepitte, D. and Moens, D. (2009) IUTAM Symposium on the Vibration Analysis of Structures with Uncertainties. Quantification of uncertain and Variable Model Parameters in Non-Deterministic Analysis. Springer Science + Business Media B. V.

[18] Telles, C.R. (2017) Methodology to Water Consumption Analysis at Schools Buildings. Parana Journal of Science and Education, 3.

[19] Sethna, J.P. (2017) Entropy, Order Parameters, and Complexity. Clarendon Press, Oxford.

[20] Kristeva, J., Rey-Debove, J. and Umiker, D.J. (1971) Essais de semiotique. Mouton, The Hague, 639 p.

[21] Coppi, R., et al. The Fuzzy Approach to Statistical Analysis. Computational Statistics \& Data Analysis, Elsevier. https://doi.org/10.1016/j.csda.2006.05.012

[22] Yates, F.E. (1994) Order and Complexity in Dynamical Systems: Homeodynamics as a Generalized Mechanics for Biology. Mathematical and Computer Modelling, 19, 49-74. https://doi.org/10.1016/0895-7177(94)90189-9

[23] Hair, J.F., et al. (2006) Multivariate Data Analysis. 6th Edition, Prentice Hall, Upper 
Saddle River.

[24] Nguyen, H.T. and Walker, E. (2000) A First Course in Fuzzy Logic. 2nd Edition, Chapman \& Hall, Boca Raton, FL, 373 p.

[25] Vicini, L. (2005) Análise multivariada da teoria à prática. UFSM, CCNE, Santa Maria, 215 p.

[26] Pedrycz, W. and Gomide, F. (1998) An Introduction to Fuzzy Sets: Analysis and Design. MIT Press, Cambridge, 465 p. 